\title{
Percepciones y prácticas relacionadas con la tuberculosis y la adherencia al tratamiento en Chiapas, México
}

\author{
Guadalupe del Carmen Alvarez-Gordillo, Dr. en C., ${ }^{(1)}$ Julio Félix Alvarez-Gordillo, Soc., ${ }^{(2)}$ \\ José Eugenio Dorantes-Jiménez, M.S.P., ${ }^{(3)}$ David Halperin-Frisch, ${ }^{\dagger}$ M.S.P. ${ }^{(I)}$
}

\section{Alvarez-Gordillo GC, Alvarez-Gordillo JF, Dorantes-Jiménez JE, Halperin-Frisch D. Percepciones y prácticas relacionadas con la tuberculosis y la adherencia al tratamiento en Chiapas, México. Salud Publica Mex 2000;42:520-528.}

\section{Resumen}

Objetivo. Conocer las percepciones y prácticas que los enfermos de tuberculosis tienen sobre la enfermedad y la adherencia al tratamiento. Material y métodos. Estudio cualitativo de II entrevistas grupales a 62 pacientes con tuberculosis diagnosticados durante 1997 y 1998 en las regiones Centro, Los Altos y Fronteriza de Chiapas, México. Resultados. Las causas de la enfermedad referidas por los pacientes fueron el contagio por trastes, el trabajo excesivo, la alimentación, el frío y otras sin relación con la transmisión de persona a persona. La incapacidad para el trabajo se reflejó en crisis económica del paciente y su familia. El estigma social impactó emocionalmente en la vida personal, familiar, laboral y de comunidad. Conclusiones. El desconocimiento sobre la enfermedad propició la elección de diferentes alternativas para su atención. Los servicios de salud y la inadecuada relación médico-paciente influyó en el retardo en el diagnóstico y falta de adherencia al tratamiento. Se sugiere un programa de difusión sobre aspectos básicos de la enfermedad y su tratamiento.

Palabras clave: tuberculosis pulmonar; adherencia al tratamiento; conocimientos, actitudes y práctica; negativa del paciente al tratamiento; relaciones médico-paciente; México

\author{
Alvarez-Gordillo GC, Alvarez-Gordillo JF, \\ Dorantes-Jiménez JE, Halperin-Frisch D. \\ Perceptions and practices \\ of tuberculosis patients and non-adherence \\ to therapy in Chiapas, Mexico. \\ Salud Publica Mex 2000;42:520-528.
}

\section{Abstract}

Objective. To identify health perceptions and practices and non-adherence to therapy among tuberculosis patients. Material and methods. Qualitative research work consisting of II group interviews with 62 tuberculosis patients during 1997-1998 in the Central, Highlands, and Border Regions of Chiapas, Mexico. Results. Perceived causes of tuberculosis included contagion via food utensils, excess work, malnutrition, and cold, as well as other causes unrelated to person-to-person contagion. The resulting incapability to work resulted in an economic crisis for both the patients and their family members. As a result of the social stigma imposed by the disease, patients perceived a negative impact on their personal life, family, work, and community. Conclusions. Lack of knowledge regarding tuberculosis is an important factor in the selection of and adherence to different care alternatives. Inadequate care provided by health services, including an unsatisfactory physician-patient relationship, resulted in diagnostic delay and non-adherence to therapy. Education programs to promote basic knowledge regarding tuberculosis and its treatment are necessary in this region.

Key words: tuberculosis, pulmonary; adherence to treatment; knowledge, attitudes, practice; treatment refusal; physicianpatient relations; Mexico

Este artículo es parte de los resultados del proyecto de investigación Adherencia al tratamiento de la tuberculosis en Chiapas. Una visión cualitativa, financiado por el Sistema de Investigación Benito Juárez (SIBEJ), Oaxaca, Oaxaca.

(I) El Colegio de la Frontera Sur, San Cristóbal de las Casas, Chiapas, México.

(2) Universidad Autónoma de Chiapas, Tuxtla Gutiérrez, Chiapas, México.

(3) Subdirección de Salud Pública, Instituto de Salud del Estado de Chiapas, Tuxtla Gutiérrez, Chiapas, México.

Fecha de recibido: 8 de noviembre de 1999 - Fecha de aprobado: 28 de septiembre de 2000 Solicitud de sobretiros: Dra. Guadalupe del Carmen Alvarez Gordillo. El Colegio de la Frontera Sur. Carretera Panamericana y Periférico Sur s/n. San Cristóbal de las Casas, Chiapas, México. Correo electrónico: galvarez@sclc.ecosur.mx 
$\mathbf{L}$ a tuberculosis continúa siendo un grave problema de salud pública. En muchos países, en la actualidad, después de que se había logrado disminuir de manera considerable el número de casos de este padecimiento por medio de programas enérgicos, se ha producido un incremento de ellos. ${ }^{1-3}$ Este cambio epidemiológico se puede atribuir, en parte, al fracaso del tratamiento no supervisado, que lleva al abandono del mismo y a la aparición de fuentes infectantes con cepas resistentes. ${ }^{4,5}$ Esto provoca que tanto los tratamientos individuales como las campañas de control sean cada vez más difíciles de implementar y de alto costo humano y social. ${ }^{6}$ La tuberculosis es la principal causa de muerte en pacientes con síndrome de inmunodeficiencia adquirida (SIDA). ${ }^{7-10}$

El diagnóstico temprano y la adherencia al tratamiento son los factores clave para el Programa Estatal de Prevención y Control de la Tuberculosis. Sin embargo, la falta de adherencia terapéutica es común con las consecuentes tasas bajas de curación. Los pacientes son usualmente diagnosticados de tuberculosis como una consecuencia de la interacción entre la búsqueda de atención y las actividades de detección pasiva de los servicios de salud. Los factores que afectan la conducta de pacientes y trabajadores de salud determinan los resultados en el diagnóstico de la enfermedad.

Este estudio ayuda a la construcción del proceso de salud-enfermedad-atención de la tuberculosis por medio de las percepciones y significaciones culturales que adquiere la enfermedad entre las diferentes personas enfermas en Chiapas. Los significados que se le atribuyen, y las maneras en que tal evento tiene lugar en una cultura determinada, influyen en el proceso de salud-enfermedad-búsqueda de atención-curación.

Se distingue de algunos modelos para explicar este proceso la perspectiva de la antropología médica que plantea una propuesta constructivista, la que considera que todo padecimiento constituye un proceso social e histórico que necesita ser reconstruido para poder comprender los significados actuales que el mismo tiene no sólo para la población sino también para el equipo de salud. ${ }^{11}$ Por lo que "según sean las condiciones de vida y de trabajo de los individuos en ciertos momentos históricos, es decir, según sea la clase y el grupo social específico al que pertenezca en una determinada formación social, será el tipo de enfermedades que se padezcan, su gravedad y su frecuencia, así como el acceso real a los servicios médicos, la esperanza de vida y de qué mueren los individuos."12,13

En este trabajo se diferenciará al padecimiento de la enfermedad, el primero, el padecimiento, como el conjunto de significaciones que tiene para un sujeto un hecho que le acontece y cuyas consecuencias para él y su entorno social son fundamentalmente negativas, y la segunda, la enfermedad, como el conjunto de signos y síntomas físicos, psicológicos, o sociales que el sujeto en el que se producen considera como tal y que es legitimado para él en alguna instancia social.

Los temas que se abordan en los resultados fueron la base del análisis y discusión bajo los siguientes ejes primordiales de la estructuración de los padecimientos. $^{14}$

1. La historia personal del sujeto, que abarca sus inserciones económicas y socioculturales como lugares en donde el padecimiento cobra significación.

2. El desencadenante manifiesto del padecimiento (la tuberculosis como enfermedad) y la vinculación con los recursos materiales y simbólicos que el sujeto utiliza para operar sobre ese desencadenante mediante prácticas y representaciones.

3. La racionalidad y valoración que el sujeto le otorga al desencadenante, al proceso y duración del padecimiento y al balance entre los beneficios (en caso de existir) y los costos y sufrimientos de ese padecimiento en un determinado momento y contexto sociocultural.

4. La legitimidad social del padecimiento -que abarca recursos, prácticas y representaciones utilizadas y ejecutadas por el sujeto- y su desencadenante para los otros significativos, así como el conflicto, ambivalencia o contradicción entre legitimidades, los que asignan los grupos sociales de pertenencia del sujeto.

5. La relación entre el padecimiento y las variables socioeconómicas del sujeto, que considera en la constitución del padecimiento lo que interviene en la articulación entre las condiciones materiales, sociales, culturales e ideológicas del sujeto y el desencadenante del padecimiento. ${ }^{14}$

Se utilizó la técnica de grupos de discusión, ya que, además, de generar su discurso correspondiente, se trabaja sobre la reducción crítica de los contenidos, mismos que se producen en un discurso grupal, que reproducen y descubren el sentido. Es así que se puede hablar del consenso, de la búsqueda de éste en la dinámica grupal. Este discurso se produce mediante discursos individuales que permiten la reflexión y se escuchan y, a su vez, son usados por los mismos participantes en forma cruzada, contrastada y enfrentada.

Esta investigación fue llevada a cabo en las regiones socioeconómicas Centro, Los Altos y Fronteriza del estado de Chiapas, con una población de 
1663473 habitantes y una densidad de población promedio de 70.6 habitantes por $\mathrm{km}^{2}$ en dichas regiones. El 49.5\% de la población está ocupada en el sector primario, $19.2 \%$ no recibe ingreso y $37.4 \%$ recibe menos de un salario mínimo. El analfabetismo es de 20\%, 38\%, $26 \%$, para las regiones Centro, Los Altos y Fronteriza, donde $6.2 \%$, $57.6 \%$ y $13.8 \%$ habla lengua indígena, respectivamente. ${ }^{15}$

En cuestión de servicios de salud, la cobertura de atención es de $93.5 \%$; en tanto $19.6 \%$ de la población está asegurada en instituciones como el Instituto Mexicano del Seguro Social (IMSS), Instituto de Seguridad y Servicios Sociales de los Trabajadores del Estado (ISSSTE) e Instituto de Seguridad Social para los Trabajadores del Estado de Chiapas (ISSTECH) y $72.7 \%$ de la población abierta recibe atención institucional de las unidades de la Secretaría de Salud (SSA) y del IMSS-Solidaridad, y sólo 1.4\% recibe atención de la medicina privada. ${ }^{*}$ Los servicios de detección, diagnóstico y tratamiento de casos del Programa Estatal de Prevención y Control de la Tuberculosis están disponibles gratuitamente en las unidades de salud de la SSA. La detección de casos es pasiva, es decir, los enfermos acuden con sintomatología respiratoria.

Los diversos factores involucrados en la presentación de la enfermedad son complejos: de los factores demográficos y sociales que se han relacionado se encuentran la gran dispersión de la población sobre el territorio (74\% habita en localidades rurales), analfabetismo, desnutrición, viviendas precarias, hacinamiento, carencia de saneamiento básico ambiental y vías de comunicación insuficientes.

Por otro lado, se ha encontrado que las percepciones culturales y las causas sociales de la enfermedad dificultan la implementación del Programa Estatal de Prevención y Control de la Tuberculosis en áreas indígenas, ya que las prácticas culturales limitan la aceptación de los servicios médicos. ${ }^{16,17}$ También se ha documentado la falta de medicamentos, insuficiente supervisión de los paramédicos en los programas y abandono de tratamiento. ${ }^{18, \ddagger}$

La eficacia del tratamiento farmacológico en cepas de Mycobacterium tuberculosis sensible a los medicamentos primarios es de $98 \% .^{19,20}$ Sin embargo, en

\footnotetext{
* Instituto de Salud del Estado de Chiapas, Dirección de Planeación, Información y Evaluación. Catálogo de Unidades Médicas, julio de 1999. Documento no publicado.

$\ddagger$ Centro de Investigaciones en Salud de Comitán. Tuberculosis and Health Care in Highland Chiapas, Mexico: An ethnographic study. Comitán Chiapas: Centro de Investigación en Salud de Comitán. Documento mimiografíado, 1991.
}

Chiapas, durante el periodo de 1989 a 1996, más de 95\% de los casos nuevos de tuberculosis pulmonar inició tratamiento y solamente $73.2 \%$ lo terminó. La eficacia fue de $90.3 \%$, lo que podría implicar la falta de supervisión del tratamiento y la presencia de cepas de $M$. tuberculosis farmacorresistentes a los medicamentos primarios. La eficiencia del tratamiento fue de $68.6 \%, 72.1 \%$, y $69.7 \%$ para las regiones Centro, Los Altos y Fronteriza, respectivamente.

\section{Material y métodos}

Se llevó a cabo un estudio cualitativo mediante entrevistas grupales a pacientes con tuberculosis de las regiones Centro, Los Altos y Fronteriza del estado de Chiapas. Se formaron 11 grupos de pacientes con tuberculosis diagnosticada durante el último trimestre de 1997 y el primer trimestre de 1998. Dos entrevistas se realizaron en un centro de salud y nueve en lugares independientes de las instituciones de salud.

Las sesiones con cada grupo tuvieron una duración de dos horas. Las entrevistas iniciaron con la presentación de los participantes y enseguida se realizaron preguntas abiertas, y se permitió que las respuestas se extendieran según las experiencias de cada uno de los participantes. Las entrevistas se grabaron, previa autorización de los pacientes, por un equipo especializado y se transcribieron por completo.

Se seleccionaron los municipios de Chiapas con mayor número de casos reportados durante 1997 y 1998. Se solicitaron los listados en las unidades de salud de la SSA, IMSS e ISSSTE. Se formaron los grupos después de visitar a cada paciente en su domicilio y de solicitar su consentimiento para participar en el estudio y darles a conocer los objetivos, métodos y beneficios del mismo.

El análisis se realizó con base en el contexto del estudio y mediante las transcripciones completas de las entrevistas grupales. Se hizo lectura en varias ocasiones de aquéllas para poder seleccionar las frases repetitivas y representativas de los pacientes y se organizaron de acuerdo con los siguientes temas: a) origen de la enfermedad; b) relación médico-paciente; c) servicios de salud; d) trabajo; e) familia; f) estigma; g) abandono; h) diagnóstico; i) tratamiento; j) educación; k) religión; l) presencia de otras enfermedades; m) cuadro clínico, y n) estrategias de control.

\section{Resultados}

El total de pacientes que participaron en las entrevistas grupales fue de 62 , con promedio de 42.4 años de edad, entre 13 y 75 años; 34 hombres y 28 mujeres. El 
$41 \%$ eran residentes de comunidades rurales y $59 \%$ de áreas urbanas. Los hombres en su mayoría trabajan en la agricultura y todas las mujeres refirieron estar dedicadas a labores del hogar, y las de medio rural colaborar además con sus esposos en labores del campo. El $90 \%$ de los pacientes que se entrevistaron estaban en la segunda fase del tratamiento para la tuberculosis (cuadro I).

Para la reconstrucción del padecimiento se abordaran los siguientes temas: a) causas de la tuberculosis; b) tuberculosis y condiciones de trabajo; c) el estigma; d) servicios de salud, y e) abandono de tratamiento. Los participantes de origen indígena no fueron diferenciados en grupos específicos, por lo que no se hizo un análisis por separado. Tampoco se detectó una variación de las percepciones y prácticas entre hombres y mujeres.

\section{Las causas de la tuberculosis}

Las causas de la enfermedad, resultado de la historia colectiva y la interacción de los enfermos en su contexto, se dividieron en siete grupos, a saber: a) contagio; b) trabajo; c) frío; d) alimentación; e) castigo de Dios; f) brujería, y g) falta a las normas.

1. Contagio. El contagio fue referido frecuentemente en relación con los trastes en los que se consumen los alimentos y ninguno comentó la transmisión de persona a persona. Dos personas preguntaron si se podía contagiar a través de las relaciones sexuales, ya que por comentarios de vecinos, se habían abstenido durante la enfermedad.

2. Trabajo excesivo. Esta relación se vinculó con la causa debido a que parte de la enfermedad trae consigo cansancio, fiebre y desgano; la persona consecuentemente adelgaza y baja de peso. Generalmente, los pacientes no pueden dejar el trabajo por depender económicamente de él. Esto trae serias dificultades a los trabajadores, porque sin ingreso económico no pueden mantener a la familia. Por ello, no pueden dejar de trabajar y por otro lado no pueden seguir un tratamiento con asistencia continua a la unidad de salud. Los pacientes hablan de las "mojadas", "del polvo", "humo", "el sol", etcétera, como condiciones desfavorables, relacionadas con los lugares del trabajo, para la aparición de la enfermedad. Lo que es obvio no es el lugar del trabajo sino el modo de vida del trabajador y su familia. En el campo la unidad productiva familiar no genera más que agricultura de subsistencia de temporal y generalmente del monocultivo de maíz. En la ciudad se encuentran pacientes de origen campesino en barrios periféricos, que debido a conflictos en sus municipios se vieron obligados a emigrar; se dedican a trabajos eventuales que ya no tienen que ver con la agricultura.

3. Frío. Fue frecuente que la enfermedad fuera relacionada también con el frío que se provoca por "descuidos" en los cambios de temperatura del cuerpo. Para el saber popular, una de las causas de la tuberculosis es mojarse, asolearse, beber frío, trabajar bajo la lluvia y bañarse. Es más notorio en un contexto rural, donde el trabajo y la mayor parte de las actividades se realizan al aire libre.

Los pacientes enfatizaron la oposición entre "frío" y "caliente" como condiciones de la sangre. Las condiciones climáticas frías y las experiencias físicas (baño frío) fueron frecuentemente ligadas a la enfermedad por esa razón.

4. La alimentación. En su mayoría los pacientes tienen deficiente alimentación por su precaria situación económica. La alimentación está basada en el maíz, frijol y chile, y aunque la alimentación no sea la causa de la enfermedad, la desnutrición contribuye a que se desarrolle. Los enfermos identifican que la mala alimentación, generalmente relacionada a la carencia de vitaminas, lleva a tener un cuerpo débil y ser propenso a dicha enfermedad.

5. Castigo de Dios. El concepto que prevalece es que "Dios causa el bien y el mal", es una voluntad omnipresente e incuestionable que lo determina todo. La concepción indígena se ha hecho permeable a la mestiza, en primer lugar por ser una herencia cultural y en segundo por el constante contacto entre ambas culturas. Por influencia de la Iglesia católica, los pacientes consideran a la enfermedad como un castigo, además de ser una dolencia acompañada de un sentimiento de culpabilidad por los pecados cometidos.

6. Brujería. Utiliza frecuentemente algún alimento que contiene algún "mal" o es provocada directamente por cuerpos extraños como los insectos (cucarachas y cienpiés), colocados por algún brujo. Por lo tanto, la curación depende de que otro brujo mediante un ritual extraiga ese "mal". El paciente mestizo comúnmente no visita al chamán o al ilól, pero existen en las ciudades importantes los llamados espiritualistas, espiritistas y hermanos, quienes curan con lociones, oraciones, hierbas y otras medicinas convencionales. Algunos pacientes recorren grandes distancias para encontrar un buen curandero. 


\section{Cuadro I \\ Características generales de los participantes en las entrevistas grupales de pacientes con tuberculosis. Chiapas, México, 1998}

\begin{tabular}{|c|c|c|c|c|c|}
\hline Grupos & $\begin{array}{l}\text { Número de } \\
\text { integrantes }\end{array}$ & $\begin{array}{l}\text { Municipios de } \\
\text { residencia }\end{array}$ & $\begin{array}{c}\text { Edad } \\
\text { promedio (años) }\end{array}$ & $\begin{array}{c}\text { Pacientes con } \\
\text { abandonos }\end{array}$ & Ocupación \\
\hline \multirow[t]{2}{*}{ Comalapa* } & 9 hombres & Comalapa, & 31.3 & I & 8 agricultores \\
\hline & & Bellavista & & & I comerciante \\
\hline \multirow[t]{3}{*}{ Comalapa* } & 6 mujeres & Comalapa, & 33.3 & 0 & 4 amas de casa \\
\hline & & Chicomuselo & & & I modista \\
\hline & & & & & I comerciante \\
\hline \multirow[t]{4}{*}{ Comalapa* } & 4 hombres & Amatenango, & 51 & I & 4 agricultores \\
\hline & I mujer & Frontera & & & I ama de casa \\
\hline & & Comalapa, & & & \\
\hline & & Chicomuselo & & & \\
\hline \multirow[t]{2}{*}{ Comitán* } & 5 mujeres & Comitán & 44.5 & I TB-MDR & 5 amas de casa \\
\hline & I hombre & & & & I velador \\
\hline \multirow[t]{4}{*}{ Comitán* } & 2 hombres & Comitán, & 55.4 & 3 & 2 comerciantes \\
\hline & 3 mujeres & Las Margaritas & & & I albañil \\
\hline & & & & & I sirvienta \\
\hline & & & & & I ama de casa \\
\hline \multirow[t]{3}{*}{ La Trinitaria* } & 3 hombres & La Trinitaria & 53.5 & 0 & 2 agricultores \\
\hline & I mujer & & & & I zapatero \\
\hline & & & & & I ama de casa \\
\hline \multirow[t]{5}{*}{ Tuxtla Gutiérrez ${ }^{\ddagger}$} & 7 hombres & Tuxtla Gutiérrez, & 34.4 & 1 & 3 estudiantes \\
\hline & & Chiapa de Corzo & & & I agricultor \\
\hline & & & & & I técnico agrario \\
\hline & & & & & I comerciante \\
\hline & & & & & I jardinero \\
\hline \multirow[t]{2}{*}{ Tuxtla Gutiérrez ${ }^{\ddagger}$} & 6 mujeres & Tuxtla Gutiérrez, & 43.5 & 2 & 5 amas de casa \\
\hline & & Chiapa de Corzo & & & I sirvienta \\
\hline Tuxtla Gutiérrez & 3 hombres & Chiapa de Corzo & 42 & 4 & 2 jardineros \\
\hline \multirow[t]{2}{*}{ Pacientes con abandonos de tratamiento } & I mujer & Tuxtla Gutiérrez & & (2 con TB-MDR) & I sirvienta \\
\hline & & & & & I agricultor \\
\hline \multirow[t]{3}{*}{ San Cristóbal de las Casas* } & 6 hombres & San Cristóbal de las Casas & 38.8 & I & 2 agricultores \\
\hline & & & & & 3 comerciantes \\
\hline & & & & & I jardinero \\
\hline \multirow[t]{2}{*}{ San Cristóbal de las Casas* } & 4 mujeres & San Cristóbal de las Casas & 53.5 & 3 & 2 comerciantes \\
\hline & & & & & 2 amas de casa \\
\hline Total & 62 & 9 municipios & 42.4 & 16 & \\
\hline \multicolumn{6}{|l|}{ * Medio rural } \\
\hline TB-MDR Tuberculosis multidrogorresis & & & & & \\
\hline
\end{tabular}


7. Falta a las normas morales y sociales. La enfermedad tiene la función sancionadora de la sociedad. La vida es aquí un escenario del hombre en constante lucha por mantener un orden o equilibrio y cualquier contratiempo es resultado de un comportamiento que no va de acuerdo con las reglas establecidas. Cuando una persona es jactanciosa o rebelde con los mayores o no cumple con la tradición es señal de que su cura sólo se hará con el cambio de actitud.

En general, para los grupos de pacientes todo abandono o desviación del comportamiento es considerado como la causa de las enfermedades.

\section{Tuberculosis y condiciones de trabajo}

La unidad económica campesina de trabajo familiar se caracteriza por la familia, equipada con medios de producción, que emplea su capacidad de trabajo en cultivar la tierra y recibe como resultado del trabajo de un año cierta cantidad de bienes. ${ }^{21}$ En el caso de campesinos con tierra tienen más posibilidades de ganancia, en el caso de la producción artesanal, bastante generalizada en Los Altos de Chiapas, es también la familia la que genera esta producción. En este contexto cualquier miembro de la familia tiene más posibilidades de sobrevivir, por la solidaridad que por la forma de producir. El problema se presenta cuando una sola persona es la responsable del ingreso económico y no tiene la propiedad de los medios de producción, es el caso de los asalariados, vendedores ambulantes, albañiles, mujeres solteras con hijos, amas de casa, choferes y jornaleros agrícolas, que son los que encontraron mayor dificultad para recibir tratamiento. Para éste fue adversa la situación de la migración por motivo de trabajo o por conflictos sociopolíticos, ya que no siempre se comunica a la institución de salud para llevar a cabo el tratamiento a donde sea factible. En unidades productivas familiares la propia familia asume la responsabilidad del paciente. Sentirse útil, necesario y querido es el motivo para tomar el tratamiento, curarse y, en consecuencia, trabajar.

La historia individual y social de los pacientes chiapanecos es una historia de dificultades, obstáculos y pobreza. La vida para ellos es un escenario donde sólo el trabajo le puede ayudar a sobrevivir. Desde la infancia la constante es el trabajo, sin poseer ningún medio de producción, largas jornadas laborales, baja escolaridad y muy bajos ingresos económicos. En la clase social de los pacientes se puede advertir que las condiciones del trabajo son causa de la enfermedad y que en la medida en que el Estado proponga solución a los bajos salarios y al desempleo habrá más posibilidades de éxito en los tratamientos y en el control de la tuberculosis.

Debido a la tuberculosis y a la importancia que le dieron a su curación, algunos pacientes dejaron de trabajar o trabajaron con grandes esfuerzos. Acudir al centro de salud significaba no darle continuidad al trabajo. La falta de ingresos necesarios para subsistir produjo en la mayoría una mayor depresión, aparte del estado físico en que ya se encontraban.

\section{El estigma de la tuberculosis}

En un contexto rural, donde las interacciones son fundamentales, la gente refiere su vida como vida en comunidad, el posible rechazo o distanciamiento de los conocidos es de un impacto muy fuerte. Por lo tanto, hombres y mujeres tratan de ocultar las manifestaciones de la tuberculosis porque temen el rechazo de sus familiares y amigos por el estigma de la enfermedad.

Una de las causas de la estigmatización de la tuberculosis fue, en muchos de los casos, el enfatizar demasiado en los cuidados, las manifestaciones exageradas por temor al contagio, porque además de dicho temor está el de que la tuberculosis sea incurable. En el contexto de lo afectivo, el contagio puede llegar a ser causa de disolver o separar a la familia, lo que constituye un factor negativo para el tratamiento. En las palabras de los pacientes mucho les ayudó la información acerca de cómo se contagia, que es curable,pero extensiva a todo el núcleo familiar, los cuidados.

El contagio como causa de la enfermedad ocasiona un ambiente de temor en torno al paciente por familiares y vecinos, que digan "ese es un tuberculoso", es un estigma o marca para el paciente y su hogar, que lo dejan de visitar, ya no le ofrecen de comer ni de beber nada, o "le buscan un traste viejo, que luego puedan tirar".

La mayoría de los pacientes conocen o han escuchado historias de personas o familias completas que han muerto de tuberculosis y que han cerrado las viviendas, por lo que la gente no se acerca a ellas pensando que podrían "contaminarse", incluso, en vida de esas personas enfermas, evitaban que "les regalaran algo y que los agarraran, menos".

\section{Servicios de salud}

A pesar de los avances de la medicina hegemónica (alópata), los enfermos conservan la visión propia, y practican una combinación de modelos de atención a la salud. Hoy, en muchas de las comunidades co- 
existen, en el orden de la tradición: el ilól, que es una persona que protege y cura las enfermedades en la comunidad; el huesero, quien también es un curandero tradicional que repara los huesos, y la partera, que atiende a las mujeres en el parto, y por otra parte, está el personal de los servicios de salud, a través de promotores comunitarios, que utilizan la medicina alópata.

Aunque todos los enfermos estaban recibiendo tratamiento farmacológico, refirieron tener algún antecedente de problema con el servicio de salud institucional, frecuentemente con el retardo en el diagnóstico; de ahí la preferencia de asistir en primera instancia a médicos particulares, que fue referida como consecuencia de algunos antecedentes de mala atención, comentarios de otras personas y en el estado grave de la enfermedad en que estuvieron algunos pacientes temieron no ser atendidos. Además de perder su día de trabajo tienen que llegar muy temprano, ya que el número de fichas y consultas son limitadas.

\section{Abandono de tratamiento}

Algunos pacientes no sabían la duración del tratamiento de la tuberculosis, por lo que la indagación sobre abandonos se realizó mediante preguntas sobre los medicamentos que habían tomado y el tiempo del propio tratamiento. Algunos no recordaban los nombres de los medicamentos, pero sí sabían que era para la enfermedad. Otros no aceptaron haber abandonado el tratamiento, justificando que sólo siguieron las instrucciones del médico.

Los pacientes que refirieron abandonar el tratamiento señalaron que al acudir a médicos particulares y debido a escasez de recursos para comprar sus medicamentos dejaron de tomarlos. Otras causas fueron las reacciones secundarias como gastritis, mareo y las molestias de aplicación en inyecciones intramusculares como el caso de la estreptomicina.

-Ni le hacía mal ni le hacía bien, entonces ya el doctor le daba una pastilla, al tomar la pastilla, haga 'sté de cuenta que una tasa de chile le dieron... se le hinchaba el estómago del dolor. La verdad es que nosotros ya no queremos ir al hospital ni al centro de salud, ni a la fuerza van hacer que entremos allí... porque estamos grandes ya, para morir de por sí tenemos que morir, ya estamos viejos y ya a la hora de una gravedad, si no puede un doctor, vamos a ver otro doctor, vamos a morir con medicina. Pues ahorita no quiero yo que lo vean la señora, porque está apesarada, que le den sus agüitas, porque está apesarada, ahorita se está declarando (Hombre, 75 años, agricultor, esposo de paciente con tuberculosis multirresistente a fármacos).
Otra de las causas detectadas fue la falta de comunicación entre el médico y el paciente, como lo ilustra otro informante que abandonó el tratamiento:

-No hallaba que hacer el doctor, él ponía aparatos, no le entendía la enfermedad... y ya por último me fui con la muchacha que curaba en Guadalupe (comunidad del municipio de Villa Las Rosas, Chiapas). Al hospital ya no volví a ir porque era muy bravo, ese rebravo, me taba metiendo aquí en la boca un fierrote así ancho y una luz, que yo abriera la boca, pero si hasta 'onde va entrar el fierro ese y estaba bien bravo, todo bravo el doctorcito, y me dijo pue «si no te componés venís otra vez» (Hombre, 65 años, albañil).

\section{Discusión}

Las percepciones y prácticas de los pacientes sobre la tuberculosis, como se demuestra en este estudio en Chiapas, intervienen en todo el proceso de saludenfermedad-atención.

En la reconstrucción del padecimiento se encuentra que la historia personal (colectiva) se refleja en las causas de la enfermedad que se inscriben y se construyen como particularidad de determinaciones estructurales colectivas relacionadas con su forma de vida y a la pobreza.

El desencadenante del padecimiento es la tuberculosis como enfermedad y a la combinatoria simultánea de medicina alópata y alternativa con el propósito de atender aspectos distintos de una misma enfermedad. Las distancias económicas, geográficas y culturales constriñen a unos a una combinatoria caracterizada por carencias y dificultades. Aquí juegan un papel muy importante los servicios de salud, en el que se reflejó la falta de comunicación del personal médico y paramédico con el paciente para transmitir la información necesaria acerca de la enfermedad y su tratamiento y como consecuencia la falta de adherencia al tratamiento.

La legitimidad social de la tuberculosis se da según el grupo de pertenencia y ámbito de la necesidad que lleva a las prácticas de cada enfermo para elegir la terapéutica, que en este estudio resultó en una combinatoria de las llamadas etnomedicinas.

En la racionalidad y valoración que el sujeto le otorga a la enfermedad es importante el estigma, la limitación al trabajo, el desequilibrio emocional al saberse enfermo y la alteración en la dinámica familiar y de pareja.

La falta de adherencia al tratamiento, en términos de la construcción social del padecimiento, no es posible predecirla, ya que estos factores se combinan en 
numerosas formas tanto como estilos de vida de cada grupo social. Un factor determinante en enfermos que utilizan la medicina alópata es la calidad del servicio de salud, principalmente en cuanto establecer una adecuada relación médico-paciente, tomando en cuenta los factores socioculturales, que según informes anteriores en otros países podrían ser una barrera mayor para el control de la tuberculosis. ${ }^{22,23}$

En el ámbito mundial se han evaluado numerosas estrategias para incrementar la adherencia al tratamiento de la tuberculosis, tales como el Tratamiento acortado estrictamente supervisado (TAES), incentivos financieros, motivación, educación y hospitalización, y son las más efectivas la supervisión diaria del tratamiento y la combinación de dos o más estrategias. $^{24-27}$

Muchas de éstas, a pesar de su éxito, tienen problema en su aplicación y su continuidad, tal como sucedió con el TAES en Chiapas, ${ }^{28}$ donde las áreas seleccionadas para esta estrategia reportan menores tasas de curación que en la totalidad del estado. ${ }^{*}$ Por lo tanto, cualquier estrategia de los programas de salud deberían ser consultadas y elaboradas en colaboración con la población objeto de las intervenciones, de manera que no se intente imponer un modelo de atención homogéneo y universal, sino responder a las necesidades características de tales grupos culturales, donde se respete su singularidad.

Los enfermos con tuberculosis aquí estudiados generaron y manejaron criterios de prevención y curación para la tuberculosis, ya que real o imaginariamente afectaron su salud en la vida cotidiana. No existen grupos que carezcan de estos saberes, porque los mismos son estructurales a toda cultura, es decir, son decisivos para la producción y reproducción de la misma. La mayoría de estos criterios preventivos son socioculturales, y no se deberían considerar como comportamientos erróneos o correctos, sino asumir que los grupos sociales producen criterios y prácticas de prevención más allá de que sean equivocados o no.

Reconocer esto supondría un cambio radical en la perspectiva salubrista, pues asumiría que los conjuntos sociales no son reacios a la prevención dado que producen y utilizan este tipo de prácticas. En consecuencia, uno de los principales objetivos médicos debería ser la descripción y el análisis de los significados de los saberes preventivos de los grupos, para trabajar

\footnotetext{
* Instituto de Salud del Estado de Chiapas. Programa Estatal de Prevención y Control de la Tuberculosis, 1999. Documento interno de trabajo.
}

a partir de los mismos en un proceso de acción conjunta salubrismo/grupos sociales. ${ }^{11}$

Por otro lado, en materia de investigación es importante generar información sobre las percepciones y prácticas del personal de salud acerca de la tuberculosis, de los enfermos y la calidad de atención que se ofrece, sin excluir a médicos particulares, a los que acuden una gran mayoría de pacientes.

\section{Agradecimientos}

Agradecemos a las antropólogas Namino Melissa Glantz, del Centro de Investigaciones en Salud de Comitán, y Dolores Molina Rosales, de El Colegio de la Frontera Sur, su colaboración en la revisión del documento, sus críticas y sugerencias que fueron de gran valor para la presentación final.

\section{Referencias}

I. Sudre P, Ten-Dam G, Kochi A. Tuberculosis a overview of the situation today. Bull World Health Organ 1991;70(2):149-159.

2. DeCock KM, Lucas SB. Global challenge of tuberculosis. Lancet 1994;344(8922):608.

3. Fleenor ME, Curtis G. Screening for tuberculosis. JAMA 1995;274(24): 1913.

4. Culliton BJ. Drug-resistant TB may bring epidemic. Nature 1992;356:473. 5. Alvarez-Gordillo GC, Halperin-Frisch D, Blancarte-Melendres L, Vázquez-Castellanos JL. Factores de riesgo para resistencia a drogas antifímicas en Chiapas, México. Salud Publica Mex 1995;37:408-416.

6. Houston S, Fanning A. Current and potential treatment of tuberculosis. Drugs 1994;48(5):689-708.

7. Riley LE, Arathoon E, Loverde VD. The epidemiologic patterns on drug resistant mycobacterium tuberculosis infections:A community-based study. Am Rev Res Dis 1989;139:1282-1285.

8. Centers for Disease Control and Prevention. Transmission of multidrug-resistant tuberculosis among immunocompromised persons, correctional system. Nueva York, 1991. JAMA 1992;268(7):855-856.

9. Edlin BR,Tokars JI, Grieco MH, Crawford JT,Williams J, Sordillo EM et al. An outbreak of multidrug-resistant tuberculosis among hospitalized patients with the acquired immunodeficiency syndrome. N Engl J Med 1992;326(23):1514-1521.

10. Small PM, Shafer RW, Hopewell PC, Singh SP, Murphy MJ. Exogenous reinfection with multidrug-resistant $M$. tuberculosis in patients with advanced HIV infection. N Engl J Med 1993;328: | |37-I | 44.

I I. Menéndez LE. Estilos de vida, riesgos y construcción social. Concepto similares y significados diferentes. Estudios Sociologicos 1998;XVI(I6): 37-67.

12. Rojas-Soriano R. Crisis, salud-enfermedad y práctica médica. México, D.F.: Plaza y Valdés 1990: 22-27.

13. Rojas-Soriano R. Sociología médica. México, D.F.: Plaza y Valdés, 1988: 7-57.

14. Módena ME. Cultura, enfermedad-padecimiento y atención alternativa. La construcción social de la desalcoholización. En: Salud, cambio social y política. México, D.F.: Edamex, 1999: 383-400. 
15. Secretaría de Hacienda y Crédito Público. Anuario Estadístico 1997. Chiapas, Tuxtla Gutiérrez, Chiapas: SHCP, 1998.

16. Menegoni I. Conceptions of tuberculosis and therapeutic choices in Highland Chiapas, México. Med Anthropol Q 1996;10(3): 38I-40I.

17. Fabrega $H$, Silver DB. Illness and shamanistic curing in Zinacantan.An ethnomedical analysis. Stanford, (CA): Stanford University Press, 1973: $8 \mathrm{I}-126$.

18. Solórzano JJ. Seguimiento de tuberculosis: estudio de 106 casos a tres años de su alta. II Congreso Nacional de Salud Pública; enero de 1991; Cuernavaca, Morelos, México.

19. Rouillon A, Pedrizet S, Parrot R, Waaler H. La transmisión del bacilo tuberculoso: el efecto de la quimioterapia. Washington, D.C.: OPS, OSP, 1977: I-28; (Publicación Científica No. 346).

20. Cano-Pérez G. Evaluación de los esquemas de tratamiento primario de tuberculosis en servicios de la Secretaría de Salud. Neumol Cir Torax 1987;XVLI(4):6-II.

21. Funes SE. Introducción a Chayanov. En: Chayanov y la teoría de la economía campesina. México, D.F.: Siglo XXI editores, 198I; Cuadernos de Pasado y Presente (94):50-53.

22. Jaramillo E. Pulmonary tuberculosis and health-seeking behaviour: How to get a delayed diagnosis in Cali, Colombia. Trop Med Int Health 1998;3(2): 138-144.
23. Jaramillo E. Tuberculosis control in less developed countries: Can culture explain the whole picture? Trop Doct 1998;28(4):196-200.

24. Volmik J, Garner P. Systematic review of randomised controlled trials of strategies to promote adherence to tuberculosis treatment. $\mathrm{Br}$ Med J 1997;315(7|20): 1403-1410.

25. Singleton L, Turner M, Haskal R, Etkind S, Tricarico M, Nardell E. Longterm hospitalization for tuberculosis control. Experience with a medical psychosocial inpatient unit. JAMA 1997;278(10):838-842.

26. Marco A, Caylá JA, Serra M, Pedro R, Sanrama C, Guerrero R, Ribot N. Predictors of adherence to tuberculosis treatment in a supervised therapy programme for prisoners before and after release. Study Group of Adherence to Tuberculosis Treatment of Prisoners. Eur Respir J 1998; 12(4):967-97I.

27. Cohen FL. Adherence to therapy in tuberculosis. Ann Rev Nurs Resp 1997;15:153-184.

28. Alvarez-Gordillo GC, Dorantes-Jiménez JE. Tratamiento acortado estrictamente supervisado para tuberculosis pulmonar en Chiapas. Salud Publica Mex 1998;40:272-275. 